\title{
Investigation of the Influence of the COVID-19 Pandemic on Medical Students' Occupational Identity
}

\author{
Siyuan Gan, Yanqin Sun*, Yanping Ha, Cailing Huang, Yanqiu Liao, Siqin Chen \\ Department of Pathology, Guangdong Medical University, Xiashan District, Zhanjiang 524023, China \\ *Corresponding author: sunyanqin@gdmu.edu.cn;
}

\begin{abstract}
Phenomenon: The COVID-19 pandemic has created great challenges for the mental health and professional identity formation of medical students in the clinic. We hope to identify the factors involved in clinical medical students' mental health and professional identity formation in the COVID-19 pandemic. Approach: We conducted a survey of professional master degree of clinical medicine students, who were enrolled in standardized residency training. The survey specifically focused on anxiety related to the COVID-19 pandemic and prevention, control, training and management measures in clinical work as well as medical students' professional identity formation during the pandemic. Findings: The pandemic had a negative impact on students' mental health. Appropriate prevention, control, training and management measures for the COVID-19 pandemic in clinical work could improve medical students' professional identity formation. The medical staff involved in anti-pandemic efforts were positive role models for medical students' professional identity formation in medical education. Insights: During the COVID-19 pandemic, it is very important to give support to students, especially those involved in clinical work, for their mental health. In addition, we should take appropriate measures for medical students' safety and to improve their professional identity formation. The medical staff involved in anti-pandemic efforts would be good role models for medical students and would contribute greatly to the formation of medical students' altruism and professional identity in medical education.
\end{abstract}

Keywords: COVID-19, Role models, Professional identity, Medical education, Medical students.

\section{Introduction}

These are unprecedented times. The spread of COVID-19 has had many adverse effects on medical education[1]. The clinical practice of medical students has been restricted, and many courses have been converted to online virtual courses[1]. Due to the lack of adequate personal protective equipment, reasonable prevention and control management measures, and adequate prevention and control training, the pandemic has not only affected medical students' clinical learning experience but also gradually affected their physical, emotional, mental well-being and professional identity formation[1,2]. Furthermore, with the spread of the pandemic, the death toll is increasing daily, and social media is flooded with negative news and discussions about the pandemic, which can worsen existing mental health problems[1].

Compared with the general population, medical students often bear a greater risk of mental stress and depression. In addition, contrary to expectations, it is more difficult for medical students to receive proper treatment and care. Because depression, the stigma of using mental health services, and access to mental health services have become obstacles to medical students' medical careers, these factors are also becoming obstacles to medical students seeking treatment[3-5]. According to a survey conducted at Changzhi Medical College in China, after the outbreak of COVID-19, $25 \%$ of 7,143 medical students were experiencing anxiety, and the level of anxiety was negatively correlated with social support[6]. During the pandemic of acute respiratory distress syndrome, a considerable number of clinical medical staff suffered obvious emotional distress. In the medical environment of the pandemic, medical students can easily become emotionally distressed and unable to cope[7]. Therefore, relieving this suffering is very important to preventing a poor mental state.

Professional identity is a complex construct linking students' motivations and abilities to their career roles. In addition, it includes students' development of professional values, actions and aspirations and their ongoing process of self-reflection on their personal identity. For medical students, professional identity is how a doctor thinks of himself or herself as a doctor. Since professional identity is critical for the acquisition of skills and knowledge relevant to patient care, it is crucial for medical education[8]. Various factors, including medical student experiences and contemporary society, affect the formation of professional identity. Additionally, medical education should respond to changes in medical student experiences and contemporary society[8]. Social, political and cultural changes cause changes in professional identity[9]. Therefore, there is no doubt that the COVID-19 pandemic would have an unprecedented impact on the professional identity formation of medical students.

In this way, the COVID-19 pandemic has created multiple challenges to the physical and mental health of medical students and their professional identity formation[1].

In China, after medical students finish their 5-year undergraduate medical education, they can choose to pursue a 3 -year graduate education program in clinical medicine, which is combined with standardized residency training. The 3 -year graduate education program in clinical medicine leads to a professional master's degree in clinical medicine[10]. Currently, this kind of educational program is one of the main training models for Chinese clinicians. There are now tens of thousands of medical students in China participating in such medical education programs. At the same time, they also participate in clinical medical work through the standardized 
residency training program.

The pandemic has already caused major changes to global education and health systems. Although people are trying their best to control the pandemic and strengthen research on treatments and vaccines, the impact of the pandemic will continue for a long time in the foreseeable future. The risk of a sudden outbreak of a pandemic always exists[11]. As the pandemic continues today, in low- and medium-risk areas, medical students have usually been undergoing inpatient and outpatient rotation as usual[12,13]. Therefore, it is critical to pay attention to the mental health of medical students in clinical learning and take effective measures to give them mental health support. At the same time, we also need to clarify what factors play an important role in the formation of medical students' professional identity during clinical learning and practice during the pandemic.

We conducted a survey of professional master's degree clinical medical students, who were enrolled in standardized residency training. The survey specifically focused on the relationship among anxiety; distress associated with the COVID-19 pandemic; prevention, control, training and management measures for the COVID-19 pandemic in clinical work; the role model status and altruism of medical staff who engaged in anti-pandemic work; and medical student professional identity formation. We aimed to identify the factors involved in clinic medical students' mental health and professional identity formation through our study. In this way, we can prepare for the impact of this unprecedented event on medical education.

\section{Method}

\subsection{Questionnaire Survey}

Questionnaires were used to evaluate professional identity and its influencing factors after the outbreak of COVID-19 among professional master's degree clinical medical students. All the students were enrolled in standard resident training. The questionnaire contained 19 questions (if the participant chose a negative answer to question 17 , then he or she answered only 18 questions). Questions 1 to 7 and question 16 were used to investigate the participants' characteristics. Questions 8 to 15 and questions 17 to 19 were Likert-scale questions designed to investigate the participants' occupational identity and their evaluations of the protection and safety of their work and study environment after the outbreak; the Likert scale ranged from 1 (strongly disagree) to 5 (strongly agree) (Table 1 ).

Table1: The content of the questionnaire.

\begin{tabular}{|c|c|}
\hline Items & Answer options \\
\hline 1. Gender & Male/Female \\
\hline \multicolumn{2}{|l|}{ 2. Age } \\
\hline 3. Grade & Year $1 / 2 / 3$ \\
\hline 5. Major & Displayed in Figure 1 \\
\hline 6. Was the major you are studying your first choice for your clinical master's degree? & Yes/No \\
\hline 7. Was medicine your first choice after you passed the college entrance examination? & Yes/No \\
\hline 8. After the outbreak of the COVID-19 pandemic, you were very worried about it. & Likert scale answer options \\
\hline $\begin{array}{l}\text { 10. The prevention, control and management measures for the COVID-19 pandemic in the daily clinical work of the } \\
\text { hospital/department where you study or work are adequate and reasonable. }\end{array}$ & Likert scale answer options \\
\hline 11. The pandemic prevention and control training of the hospital/department where you study or work are appropriate. & Likert scale answer options \\
\hline 12. The hospital/department where you study or work has sufficient protective materials. & Likert scale answer options \\
\hline 13. Are there any medical staff among your relatives? & Yes/No \\
\hline $\begin{array}{l}\text { 14. After the outbreak of the COVID-19 pandemic, your enthusiasm and sense of mission in clinical medicine have greatly } \\
\text { increased. }\end{array}$ & Likert scale answer options \\
\hline $\begin{array}{l}\text { 15. Since the outbreak of the COVID-19 pandemic, a great number of the heroic deeds of the anti-pandemic workers have } \\
\text { greatly helped you to strengthen your ideals of practicing medicine and increase your sense of mission to heal the wounded and } \\
\text { rescue the dying. }\end{array}$ & Likert scale answer options \\
\hline $\begin{array}{l}\text { 16. Are there any medical staff who went to Hubei to fight against the COVID-19 pandemic among your teachers, friends or } \\
\text { relatives in } 2020 \text { ? }\end{array}$ & Yes/No \\
\hline $\begin{array}{l}\text { 17. In } 2020 \text {, teachers, friends or relatives who went to Hubei to fight against the COVID- } 19 \text { pandemic acted bravely and } \\
\text { fearlessly. This will greatly help you to strengthen your medical ideals and enhance your sense of mission in clinical work. }\end{array}$ & Likert scale answer options \\
\hline
\end{tabular}
Strongly Disagree).

We conducted a cross-sectional survey from January 16, 2021, to January 25, 2021.

Before the start of the survey, all participants were informed of the research purpose and content of the survey; in addition, they were informed that this survey was voluntary and that they could stop their participation in the study at any time. All participants in this study participated anonymously, and the data of individual participants could not be traced in the results. The Institutional Ethical Review Board of the affiliated hospital of Guangdong Medical University confirms that ethics approval was not required. The questionnaire was administered through the Questionnaire Star online survey tool. Participants could click the link to open the questionnaire on a computer and then complete it, or they could scan the questionnaire QR code through the WeChat App to complete it using a mobile phone.

\subsection{Statistical Analysis}

Participants' responses to questionnaire items 8, 9, 10, 11, 12, $14,15,18$, and 19 were analyzed using $t$ tests to determine the differences between those with or without relatives who were medical staff, as well as between those with or without teachers, relatives or friends who had been to Hubei to participate in the anti-pandemic work. The correlations 
among questionnaire items 8, 9, 10, 11, 12, 14, 15, 18, and 19 were analyzed using Pearson correlation analyses. A p-value $<0.05$ was considered statistically significant. GraphPad Prism 7 (GraphPad software, La Jolla, CA, USA) was used for Pearson correlation analyses and t tests.

\section{Results}

There were 377 participants, including 201 women and 176 men (Figure $1 \mathrm{~A}$ ). The youngest participant was 20 years old, and the oldest was 44 years old. There were 62 people in the $20-23$ age group, 287 people in the $24-27$ age group, and 28

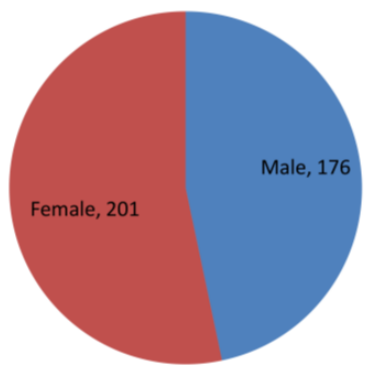

A

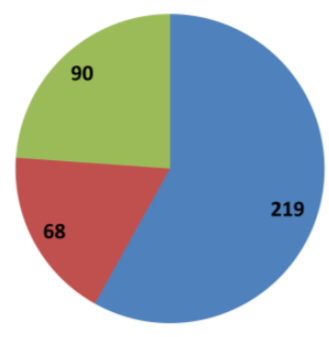

- year 1
- year 2

\pm year 3

C people in the 28-44 age group (Figure $1 \mathrm{~B}$ ). Among the participants, there were 219 in their first year, 68 in their second year, and 90 in their third year (Figure 1C). A total of 280 survey participants reported that the major they were studying was their first choice, while 97 participants said it was not. A total of 339 survey participants indicated that medicine was their first choice after the college entrance examination, while 38 participants said it was not. A total of 182 participants had relatives who were medical staff, but 195 participants did not. A total of 176 participants had teachers, relatives or friends who had been to Hubei to participate in the anti-pandemic work, but 201 participants did not (Figure 1D).

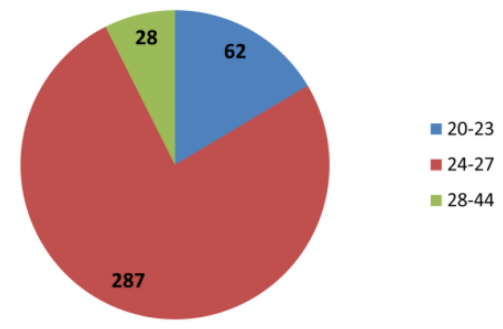

B

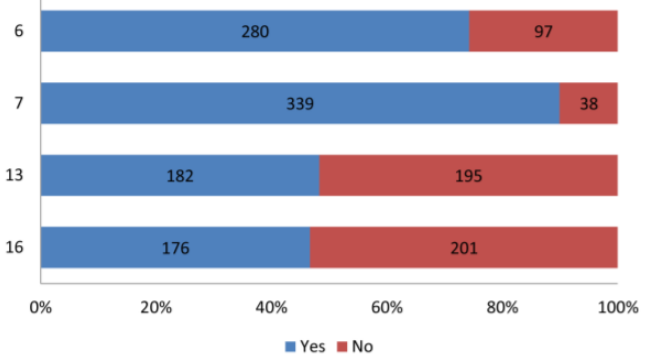

D

Figure 1: Group characteristics of the questionnaire survey participants $(n=377)$

Notes: A, Gender characteristics of the participants; B, Age characteristics of the participants; C, Grade characteristics of the participants; D, Participants' answers to questions $6,7,13,16$. The full descriptors are displayed in Table 1 . The numbers of respondents are shown in the corresponding areas.

The survey participants were from 7 universities (Figure $2 \mathrm{~A}$ ). Their majors were widely distributed, with 39 people studying respiratory medicine, infectious diseases or emergency medicine, which are most closely related to COVID-19. On the other hand, there were 65 people studying other internal medicine majors and 95 people studying surgery majors (Figure $2 \mathrm{~B}$ ).

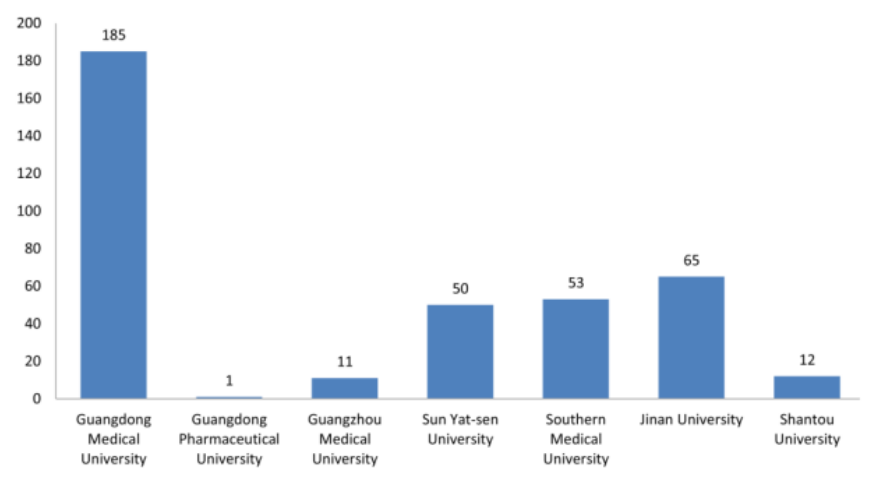

A

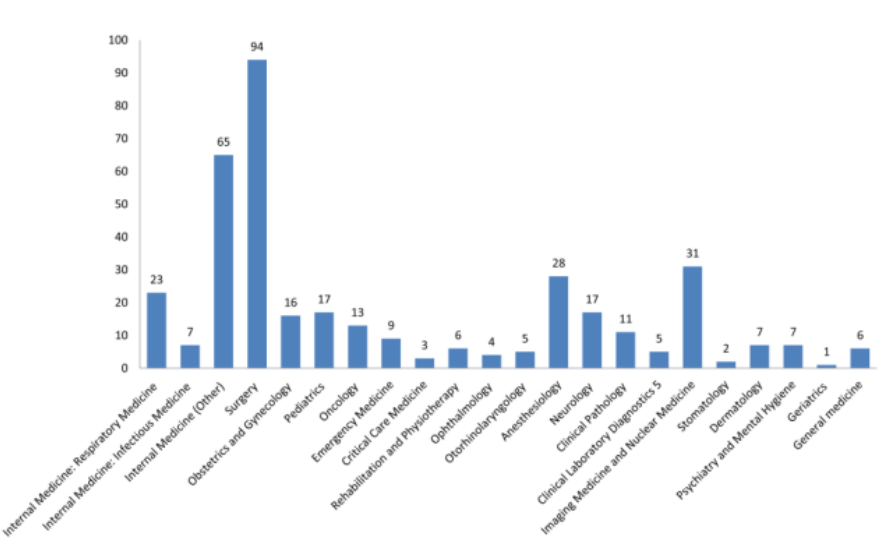

B

Figure 2: Participants' school and major distributions $(n=377)$

Notes: A, Participants' school distribution; B, Participants' major distribution. 
More than half of the students strongly agreed or agreed that after the outbreak of the COVID-19 pandemic, they were very worried about the pandemic or were very worried about their own safety in clinical work. Most of the students strongly agreed or agreed that the prevention, control and management measures for the COVID-19 pandemic in daily clinical work and the pandemic prevention and control training of the hospital/department where they studied or worked were adequate and reasonable. In addition, most of the students strongly agreed or agreed that there were sufficient protective materials in the hospital/department where they studied or worked. Meanwhile, most of the students strongly agreed or agreed that after the outbreak of the COVID-19 pandemic, their enthusiasm and sense of mission in clinical medicine greatly increased and that the heroic deeds of the anti-pandemic workers greatly helped them to strengthen their ideals of practicing medicine and increase their sense of mission to heal the wounded and rescue the dying. Furthermore, most of the students strongly agreed or agreed that they were looking forward to becoming a clinician and were proud of being a young clinician (Figure 3).

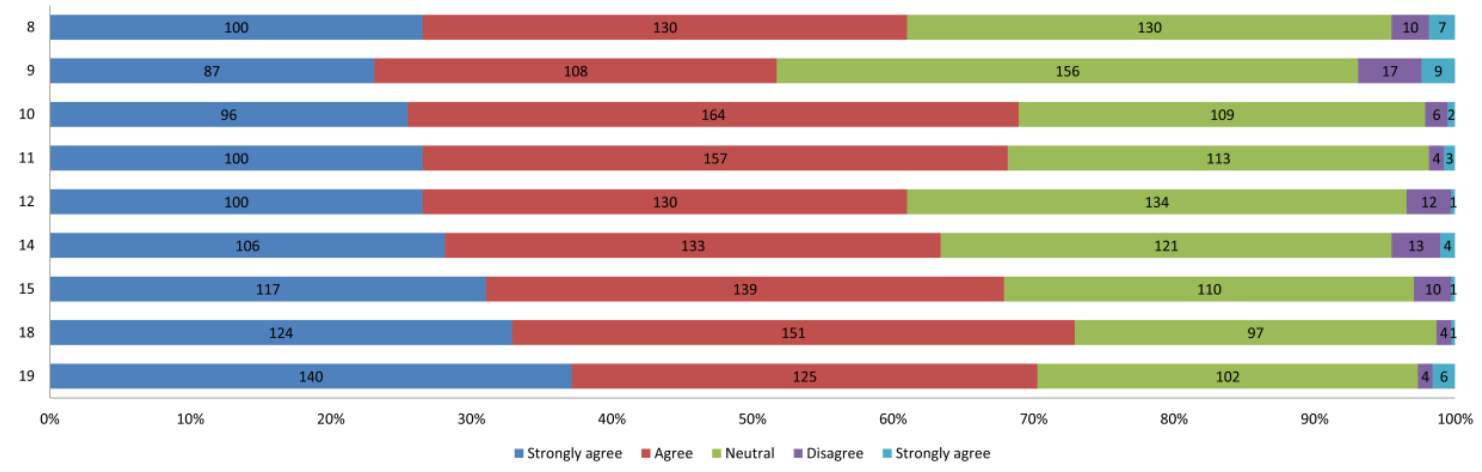

Figure 3: Students' perception of their safety during the COVID-19 pandemic, professional identity, recognition for anti-pandemic workers, and prevention of the COVID-19 pandemic $(n=377)$.

Notes: The 9 items in the questionnaire were rated on a scale from strongly disagree to strongly agree. The full descriptors are displayed in Table 1 . The numbers of respondents are shown in the corresponding areas.

The students' concerns about the pandemic were closely related to their concerns about the safety of clinical work (Figure 4, $\mathrm{P}<0.01$ ). There were significant positive correlations among the prevention, control and management measures for the COVID-19 pandemic in daily clinical work; pandemic prevention and control training in the hospital/department; and sufficient protective materials in the hospital/department where the students studied or worked (Figure 4, P<0.01). Furthermore, after the outbreak of the COVID-19 pandemic, students' enthusiasm and sense of mission in clinical medicine were significantly positively associated with prevention, control and management measures for the COVID-19 pandemic in daily clinical work; pandemic prevention and control training in the hospital/department; and sufficient protective materials in the hospital/department where the students studied or worked (Figure 4, $\mathrm{P}<0.01$ ). There were significant positive correlations among the students' enthusiasm and sense of mission in clinical medicine; the strengthening of the students' ideals of practicing medicine and sense of mission to heal the wounded and rescue the dying due to the heroic deeds of the anti-pandemic workers; the students' enthusiasm for becoming a clinician to heal the wounded, rescue the dying and serve society; and the students' pride in being a young clinician (Figure 4, $\mathrm{P}<0.01$ ). Furthermore, compared with the students with no medical staff relatives, the students with medical staff among their relatives were more likely to report a enthusiasm and sense of mission in clinical medicine; the strengthening of their ideals of practicing medicine and sense of mission to heal the wounded and rescue the dying due to the heroic deeds of the anti-pandemic workers; their enthusiasm for becoming a clinician to heal the wounded, rescue the dying and serve society; and their pride in being a young clinician (Figure 5, P<0.01). Students with teachers, relatives or friends who had been to Hubei to fight the COVID-19 pandemic reported greater satisfaction than students with no teachers, relatives or friends who had been to Hubei to fight the COVID-19 pandemic with the prevention, control and management measures for the COVID-19 pandemic in daily clinical work; pandemic prevention and control training in the hospital/department; and protective materials in the hospital/department (Figure 6, $\mathrm{P}<0.05$ ). Compared with those without teachers, relatives or friends who had been to Hubei to fight against the COVID-19 pandemic, students with such teachers, relatives, or friends were more likely to report their enthusiasm and sense of mission in clinical medicine; the strengthening of their ideals of practicing medicine and sense of mission to heal the wounded and rescue the dying due to the heroic deeds of the anti-pandemic workers; their enthusiasm for becoming a clinician to heal the wounded, rescue the dying and serve society; and their pride in being a young clinician (Figure 6, $\mathrm{P}<0.01)$

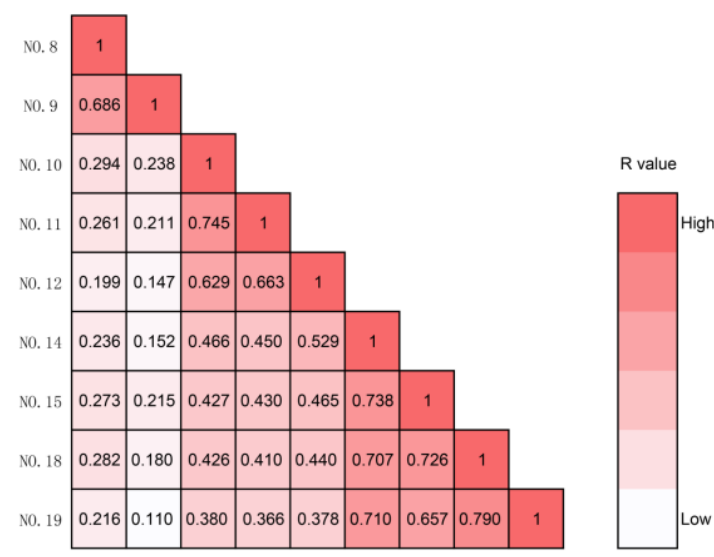

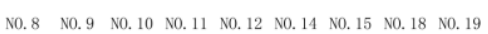

Figure 4: Correlation among professional identity, recognition of anti-pandemic workers and prevention of the COVID-19 pandemic

Notes: Analyzed by Pearson correlation $(\mathrm{P}<0.05$ or $\mathrm{P}<0.01)$. The figure in the grid is the $R$ value. 


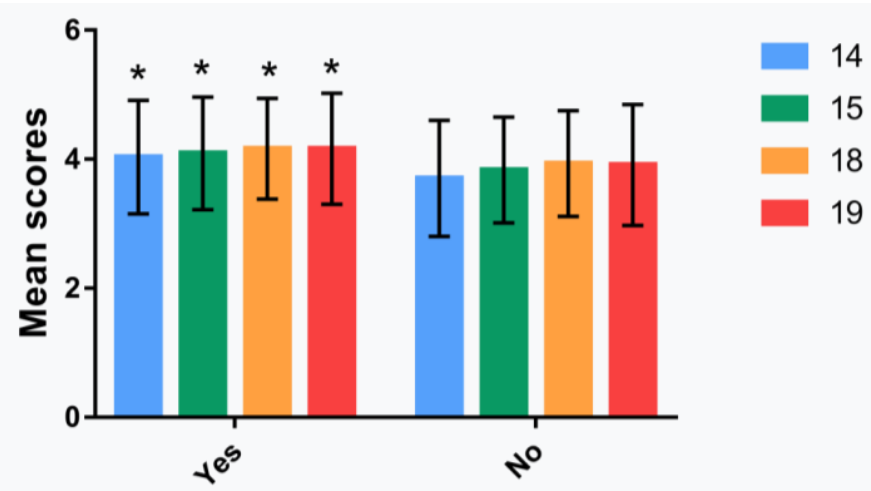

Figure 5: Comparisons of professional identity and recognition of anti-pandemic workers between students with and without relatives who were medical staff (mean \pm SD)

Notes: Analyzed by one-way ANOVA. Yes: the group with relatives who were medical staff ( $\mathrm{n}=182 /$ group), No: the group without relatives who were medical staff ( $\mathrm{n}=195 /$ group). The full descriptors of questions $14,15,18$, and 19 are displayed in Table $1 . * \mathrm{P}<0.01$, compared with the group without relatives who were medical staff.

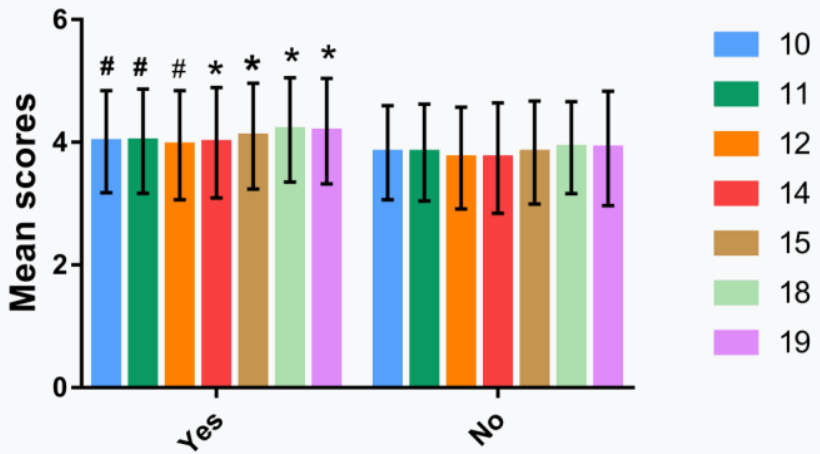

Figure 6: Comparisons of professional identity, recognition of anti-pandemic workers, and prevention of the COVID-19 pandemic between students with and without teachers, relatives or friends who had been to Hubei to fight against COVID-19 pandemic (mean $\pm \mathrm{SD})$

Notes: Analyzed by one-way ANOVA. Yes: the group with teachers, relatives or friends who had been to Hubei to fight against COVID-19 pandemic ( $n=176 /$ group), No: the group without teachers, relatives or friends who had been to Hubei to fight against COVID-19 pandemic ( $n=201 /$ group). The full descriptors of questions $10,11,12,14,15,18$, and 19 are displayed in Table 1 . $\# \mathrm{P}<0.05, * \mathrm{P}<0.01$, compared with the group without teachers, relatives or friends who had been to Hubei to fight against COVID-19 pandemic.

Most of the students strongly agreed or agreed that having friends or relatives who had been to Hubei to fight against COVID-19 pandemic who acted bravely and fearlessly greatly strengthened their medical ideals and enhanced their sense of mission in clinical work (Figure 7).

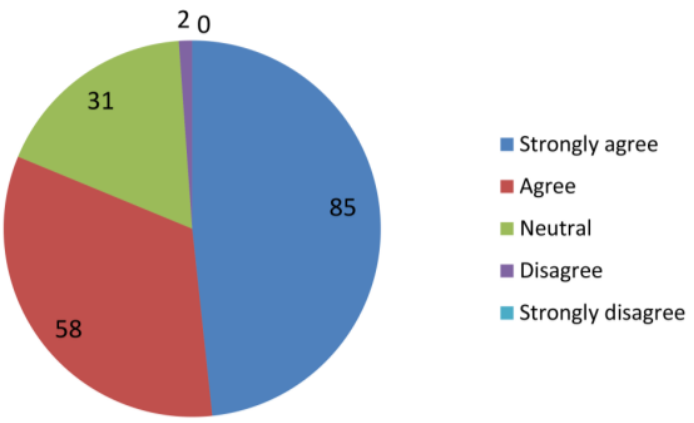

Figure 7: The perceptions of respondents with relatives or friends who had been to Hubei to participate in the anti-pandemic work $(\mathrm{n}=176)$.

Notes: Question 16 was rated from strongly disagree to strongly agree. The full descriptors are displayed in Table 1. The numbers of respondents are shown in the corresponding areas.

\section{Discussion}

The professional master's degree clinical medical students were worried about the pandemic and their own safety in clinical work, and their concerns about the pandemic were closely related to their concerns about their own safety in clinical work. Meanwhile, we found that a considerable number of participants admitted that they were greatly concerned or concerned about the pandemic and that they were very worried or worried about their own safety in clinical practice. This finding indicates that the COVID-19 pandemic has caused great troubles for the mental and psychological health of students. A prospective longitudinal study demonstrated that the COVID-19 pandemic had a negative impact on the mental health of medical students. The pandemic significantly increased the prevalence and levels of anxiety and stress[14]. A study analyzed the effects of COVID-19 crisis-related stressors on residents' professional and personal lives. The study demonstrated that anxiety about personal protective equipment shortages was significantly associated with increased stress during the COVID-19 pandemic[15]. Overall, our study demonstrates that during the COVID-19 pandemic, we must pay attention to the mental health of students, especially those involved in clinical work, and take practical measures to give students mental and psychological support. However, our study found that there were no correlations between students' concerns and anti-pandemic prevention and control measures, materials, management and training or professional identity formation $(\mathrm{R}<0.4)$. Furthermore, whether any relatives were medical staff and whether anyone around them had been to Hubei to participate in the fight against the pandemic had no impact on their concerns. This finding suggests that further research is needed to identify the factors that affect students' concerns 
and professional identity information.

In addition, a survey was conducted to study UK medical students' willingness to be placed in clinical environments during the COVID-19 pandemic. Most of the participants indicated that they were willing to participate in clinical internships, and most people in this group were willing to be placed in wards, even though they felt that it would not be a safe clinical environment for them. A number of responses have called for clearer guidelines from medical schools. In addition, instructions regarding the appropriate use of protective equipment are also required. Most people in this study $(80.6 \%, \mathrm{n}=166)$ reported that medical schools had taken measures to reduce their risk of exposure to the virus[16]. Our study found that good prevention and control measures, adequate protective equipment, reasonable pandemic management and prevention and control training are mutually reinforcing. In addition, these factors can enhance professional identity. Therefore, we should take appropriate prevention and control measures, provide adequate protective equipment and establish reasonable pandemic management and prevention and control training to effectively prevent the spread of the pandemic; protect students, medical staff, and patients; and improve the professional identity of medical students.

Students' experiences in the educational system significantly impact their own professional identity formation. Physicians who internalize their professional roles are greatly affected by the medical training model[17]. In an ideal teaching model, students are learners who need supervision. The formation of students' professional identity occurs through role modeling in the teaching process; that is, they learn to prioritize patients and aspire to altruism[12]. Interactions with other healthcare professionals and patients are critical for medical students' professional identity formation[8]. In previous crises, such as fires, earthquakes or terrorist attacks, medical students could still continue their education and clinical training. They could not only take on social responsibilities and make contributions to responding to crises but also enhance their professional identity in the process[12]. However, in the pandemic context, medical students cannot participate in the care of COVID-19 patients due to the danger of infection and transmission of the virus, the lack of personal protective equipment and other restrictions. Furthermore, even in the early stages of the pandemic, clinical internships and learning were suspended and could only be replaced by remote virtual teaching courses[12,13]. This change will undoubtedly affect the formation of students' professional identity[12].

Previous studies have found that role models contribute greatly to medical students' professional identity formation[18,19]. Furthermore, positive role models not only are the most important factor for professional identity formation but also are critical to the psychological well-being of medical students[20,21]. In the early stage of the outbreak in 2020, when Hubei's medical system was overwhelmed, tens of thousands of medical staff from across the country went to Hubei to work together to fight against the pandemic[22]. Facing this unprecedented public health challenge, staff and volunteers from the National Centers for Disease Control, big data centers at multiple levels, primary medical institutions, general hospitals and designated hospitals, and government agencies participated in the overall response[22]. The medical staff who played a key role in responding to this crisis are undoubtedly role models recognized and respected by the general public, including medical students. In our study, the students with medical staff among their relatives and friends had a better professional identity, greater recognition of the deeds of anti-pandemic workers, and greater recognition that the deeds of anti-pandemic workers were effective in promoting their professional identity. During the pandemic, students with relatives and friends who had been to Hubei to fight the pandemic were more likely to recognize that their working environment had good prevention and control measures, adequate protective equipment, reasonable pandemic management and prevention and control training, and better professional recognition. Furthermore, they were more likely to recognize the deeds of anti-pandemic workers and that these deeds more effectively promoted their professional recognition. This demonstrates that the students believed that the medical staff role models around them, especially those who had been to Hubei to participate in the fight against the pandemic, greatly promoted the formation of their altruism and professional identity during the COVID-19 pandemic.

As mentioned above, medical students' experiences in the medical education system directly affect their professional identity formation. Therefore, in response to unprecedented challenges during the pandemic, medical education, especially medical ethics education related to the formation of professional identity and the humanities education curriculum, must be reformed accordingly. However, under the guidance of medical ethics and medical ethics, efforts to solve medical practice problems and social problems during the pandemic are still relatively insufficient. At present, the research and attention on these issues in Chinese academic circles is still insufficient. Whether it is in undergraduate teaching courses, postgraduate teaching courses or continuing education, medical ethics education is often marginalized and not taken seriously. To solve this problem of insufficient preparation, we should explore new ways and new focuses of medical ethics teaching in the context of the pandemic[22].

Studies conducted at the University of Haifa in Israel evaluated the effectiveness of bioethics courses in medical schools, and the results showed that it is difficult for course teaching to achieve good translation effects in the clinic[23]. Based on the results of this research, researchers have launched a new teaching model that vertically integrates the horizontal medical ethics curriculum into the entire medical curriculum in several medical schools in India and that has achieved good results[23]. A previous study suggested that clear goals are crucial for students to be taught or for students to learn to be excellent medical professionals[24]. Therefore, the medical staff involved in the fight against the pandemic can act as role models for medical students and residents. We should encourage the medical staff involved in the fight against the pandemic to devote themselves to medical education in various forms and vertically integrate their own experiences into undergraduate and graduate medical ethics curricula and even the entire medical curriculum by narrating their experiences. In addition, role modelling should not be limited to classroom lectures. Schools and hospitals should provide a variety of teaching models, such as informal chat 
sessions, case discussions, handover meetings, and seminars.

\section{Conclusion}

During the COVID-19 pandemic, it is very important to give support to medical students, especially those involved in clinical work, for their mental health. In addition, we should take appropriate prevention and control measures, provide adequate protective equipment and reasonable pandemic management and prevention and control training to improve medical students' safety and professional identity formation. Furthermore, the medical staff involved in anti-pandemic efforts would be good role models for medical students. These role models could contribute greatly to the formation of medical students' altruism and professional identity in medical education. Therefore, we should play their role as role models to improve students' professional identity and altruism formation.

\section{Acknowledgement}

This work was supported by the Zhanjiang City Non-funded Science and Technology Research Project (NO. 2021B01043 and 2020B01147), Guangdong Postgraduate Education Innovation Program (NO.2019JGXM56), Guangdong Higher Education Reform Program (NO.2018270); Guangdong Education Science "13th-five year plan" Research Project of 2020 (NO.2020GXJK412), Guangdong Province Educational Science Planning Project of 2021 (Higher Education Special Project) (NO. 2021GXJK468), Teaching Quality and Teaching Reform Project of Guangdong Medical University(NO. 1JG21096), The "14th Five-Year Plan" of Guangdong Higher Education Association Research Project for Higher Education in 2021 (NO.21GYB158), Guangdong Medical University Special Project of Course Ideological and Political Education Teaching Research of 2021 (NO.2SZ21020)

\section{Conflict of Interest Disclosure}

The authors report no conflicts of interest. The authors alone are responsible for the content and writing of this article.

\section{References}

[1] S. Chandratre, "Medical students and COVID-19: challenges and supportive strategies," J Med Educ Curric Dev, 7, pp.2382120520935059, 2020.

[2] A. Rastegar Kazerooni, "Peer mentoring for medical students during the COVID-19 pandemic via a social media platform," 54(8), pp.762-763, 2020.

[3] T. L. Schwenk, "Depression, stigma, and suicidal ideation in medical students," JAMA, 304(11), pp.1181-1190, 2010.

[4] J. Tjia, "Factors associated with under treatment of medical student depression," J Am Coll Health, 53(5), pp.219-224, 2005.

[5] J. L. Givens, "Depressed medical students' use of mental health services and barriers to use," Acad Med, 77(9), pp.918-921, 2002.
[6] W. Cao, "The psychological impact of the COVID-19 epidemic on college students in China," Psychiatry Res, 287, pp.112934, 2020.

[7] L. O'Byrne, "Medical students and COVID-19: The need for pandemic preparedness," 46(9), pp.623-626, 2020.

[8] I. Wilson, "Professional identity in medical students: pedagogical challenges to medical education," Teach Learn Med, 25(4), pp.369-373, 2013.

[9] F. Consortium, "Decomposing and recomposing occupational identities-A survey of theoretical concepts," Springer Netherlands, 2007.

[10] Q. Zhang, "Medical education: Changes and perspectives," Med Teach, 35(8), pp.621-627, 2013.

[11] A. Al Samaraee, "The impact of the COVID-19 pandemic on medical education," Br J Hosp Med (Lond), 81(7), pp.1-4, 2020.

[12] S. Rose, "Medical student education in the time of COVID-19," JAMA, 323(21), pp.2131-2132, 2020.

[13] V. Kaul, "Medical Education during the COVID-19 Pandemic," Chest, 159(5), pp.1949-1960, 2021.

[14] I. Saraswathi, "Impact of COVID-19 outbreak on the mental health status of undergraduate medical students in a COVID-19 treating medical college: a prospective longitudinal study," 8, pp.e10164, 2020.

[15] T. Rana, "Medicine and surgery residents' perspectives on the impact of COVID-19 on graduate medical education," Med Educ Online, 25(1), pp.1818439, 2020.

[16] J. J. Park, "Medical student perceptions of working in clinical environments during the COVID-19 pandemic,' J Glob Health, 10(2), pp.020380, 2020.

[17] M. D. Beaulieu, "Family practice: Professional identity in transition: A case study of family medicine in Canada," Soc Sci Med, 67(7), pp.1153-1163, 2008.

[18] J. Goldie, "Teaching professionalism in the early years of a medical curriculum: a qualitative study," Med Educ, 41(6), pp.610-617, 2007.

[19] M. T. White, "Perceptions of factors contributing to professional identity development and specialty choice: A survey of third- and fourth-year medical students," Annals of Behavioral Science and Medical Education, 17(1), pp.18-23, 2011.

[20] A. Baernstein, "Learning professionalism: perspectives of preclinical medical students," Acad Med, 84(5), pp.574-581, 2009

[21] M. J. Cohen, "Identity transformation in medical students," Am J Psychoanal, 69(1), pp.43-52, 2009.

[22] D. Y. Yang, "Preparedness of medical education in China: Lessons from the COVID-19 outbreak," Med Teach, 42(7), pp.787-790, 2020.

[23] R. F. D'Souza, "Novel horizontal and vertical integrated bioethics curriculum for medical courses," 40(6), pp.573-577, 2018.

[24] A. C. Schwartz, "Developing a modern standard to define and assess professionalism in trainees," Acad Psychiatry, 33(6), pp.442-450, 2009. 